\title{
The Variable Separation Method and Exact Jacobi Elliptic Function Solutions for the Nizhnik-Novikov-Veselov Equation
}

\author{
Y.-Z. PENG* \\ Department of Mathematics \\ Huazhong University of Science and Technology \\ Wuhan, 430074, P.R. China
}

(Received October 10, 2005; revised version January 31, 2006)

\begin{abstract}
Based on the singular structure analysis, the variable separation method is proposed for the Nizhnik-Novikov-Veselov equation to obtain a general functional separation solution containing three arbitrary functions. Choosing these arbitrary functions to be the Jacobi elliptic functions, a diversity of elliptic function solutions may be obtained for the equation of interest. The interaction property of the waves is numerically studied. The long wave limit gives the new type of localized coherent structures.
\end{abstract}

PACS numbers: 02.30.Jr

\section{Introduction}

If a given nonlinear partial differential equation (PDE), say, in two independent variables

$$
P\left(x, t, u, u_{x}, u_{t}, \ldots\right)=0,
$$

admits a nontrivial solution of the form

$$
\begin{aligned}
& u=f(w), \\
& w=a(x)+b(t),
\end{aligned}
$$

we talk of functional separation [1]. The classical additive separable solution $u=a(x)+b(t)$, or product separable solution $u=a(x) b(t)$, are particular cases of functional separation. A simple particular case of (2) is the travelling wave solution, which is very well understood [2] and hence omitted. Some authors [3-6] consider the functional separable solutions of nonlinear PDEs based on the general

*e-mail: yanzepeng@163.com 
ansatz (2). From the point of view of symmetry group, two methods have been proposed to study the compatibility of the ansatz (2) and Eq. (1) in question. One is the nonclassical method [7] where three different invariant surface conditions are used to characterize the functional separable solutions. The other is the generalized conditional symmetry approach based on the ansatz (2) [8]. In this paper, we study the functional separable solutions of nonlinear PDEs based on the singular structure analysis. Especially, we use the Nizhnik-Novikov-Veselov (NNV) equation as a simple illustration example.

The organization of the paper is as follows. In Sect. 2, a general functional separation solution containing three arbitrary functions is obtained for the equation of interest. By choosing these arbitrary functions to be the Jacobi elliptic functions, a class of elliptic function solutions are given in Sect. 3. Section 4 is devoted to studying the interaction properties of waves by virtue of graphic method. Some conclusion and discussion are given in the last section.

\section{The variable separation method for the NNV equation}

The Nizhnik-Novikov-Veselov equation

$$
\begin{aligned}
& u_{t}+a u_{x x x}+b u_{y y y}-3 a(u v)_{x}-3 b(u w)_{y}=0, \\
& u_{x}=v_{y}, \\
& u_{y}=w_{x},
\end{aligned}
$$

is an isotropic Lax integrable extension of the $(1+1)$-dimensional Korteweg-de Vries (KdV) equation [9, 10]. Boiti et al. [11] have solved the NNV equation by means of the inverse scattering transformation method and Radha et al. [12] obtained its multidromion solution. The physical importance of Eq. (3) can be easily found in Refs. [9-12]. However, the NNV equation possesses many interesting solution structures which have not yet been found. By means of the singular structure analysis [13], we obtain the following transformation:

$$
\begin{aligned}
& u=\frac{2 \varphi_{x} \varphi_{y}}{\varphi^{2}}-\frac{2 \varphi_{x y}}{\varphi}, \\
& v=\frac{2 \varphi_{x}^{2}}{\varphi^{2}}-\frac{2 \varphi_{x x}}{\varphi}+v_{2}(x, t), \\
& w=\frac{2 \varphi_{y}^{2}}{\varphi^{2}}-\frac{2 \varphi_{y y}}{\varphi}+w_{2}(y, t),
\end{aligned}
$$

where the singular manifold $\varphi$ satisfies

$$
\begin{aligned}
& \varphi_{t}+a \varphi_{x x x}+b \varphi_{y y y}-3 a v_{2} \varphi_{x}-3 b w_{2} \varphi_{y}=0, \\
& a \varphi_{x} \varphi_{x x x y}+b \varphi_{y} \varphi_{x y y}-a \varphi_{x x x} \varphi_{x y}-b \varphi_{x y} \varphi_{y y y}=0, \\
& a \varphi_{x} \varphi_{x x} \varphi_{x y}+b \varphi_{y} \varphi_{y y} \varphi_{x y}-a \varphi_{x}^{2} \varphi_{x x y}-b \varphi_{y}^{2} \varphi_{x y y}=0,
\end{aligned}
$$

with $v_{2}$ and $w_{2}$ being two arbitrary functions of indicated variables. It is easily 
checked that Eq. (5) has the additive separable solution

$$
\varphi=f(x, t)+g(y, t)
$$

if

$$
\begin{aligned}
& v_{2}=\frac{f_{t}+a f_{x x x}-\lambda(t)}{3 a f_{x}}, \\
& w_{2}=\frac{g_{t}+b g_{y y y}+\lambda(t)}{3 b g_{y}},
\end{aligned}
$$

where $f(x, t), g(y, t)$ and $\lambda(t)$ are arbitrary functions of indicated variables. The substitution of Eqs. (6) and (7) into Eq. (4) yields a general functional separation solution of the NNV equation (3)

$$
\begin{aligned}
& u=\frac{2 f_{x} g_{y}}{(f+g)^{2}}, \\
& v=\frac{2 f_{x}^{2}}{(f+g)^{2}}-\frac{2 f_{x x}}{f+g}+\frac{f_{t}+a f_{x x x}-\lambda(t)}{3 a f_{x}}, \\
& w=\frac{2 g_{y}^{2}}{(f+g)^{2}}-\frac{2 g_{y y}}{f+g}+\frac{g_{t}+b g_{y y y}+\lambda(t)}{3 b g_{y}},
\end{aligned}
$$

with $f(x, t), g(y, t)$ and $\lambda(t)$ being arbitrary functions of indicated variables. Let us note that the solution formula (8) has been already obtained by $\mathrm{Hu}$ et al. [14] by the Darboux transformation. From Eq. (8) one can see that the NNV equation possesses localized coherent structures for the physical field $u$ rather than the potential field $v$ and $w$, as pointed out in Ref. [12]. Therefore the physical field $u$ alone is given in the following.

\section{The exact elliptic function solutions of Eq. (3)}

It is due to the arbitrariness of the functions $f, g$, and $\lambda$ that Eq. (8) displays an interesting variety of solution structures. In this paper, we are restricted to Jacobi elliptic function solutions. It is worth noticing that the Jacobi transformation $\operatorname{dn}(\xi \mid m)=\operatorname{cn}\left(\sqrt{m} \xi \mid m^{-1}\right)$ [15] implies that any solution found by the dn-function may be transformed into an equivalent one that can be obtained by cn-function. Moreover, since other Jacobi elliptic functions have singularities, we consider only elliptic function solutions in terms of sn- and cn-functions. Several cases are considered.

Case 1. $f=\operatorname{sn}\left(k x-\omega_{1} t \mid m_{1}\right) \equiv \operatorname{sn} \xi, g=\operatorname{sn}\left(l y-\omega_{2} t \mid m_{2}\right)+A \equiv \operatorname{sn} \eta+A$.

From Eq. (8), we obtain the exact elliptic function solution to Eq. (3)

$$
u=\frac{2 k l \operatorname{cn} \xi \operatorname{dn} \xi \operatorname{cn} \eta \operatorname{dn} \eta}{(\operatorname{sn} \xi+s n \eta+A)^{2}}
$$

where $k, l, \omega_{1}, \omega_{2}$, and $A$ are arbitrary constants, $m_{1}$ and $m_{2}$ the moduli of the elliptic functions, and the constant $A$ must be greater than 2 to guarantee 
that the solution has no singularity (because sn- and cn-functions have zeros, $\min (\operatorname{sn} \xi+\operatorname{sn} \eta)=-2$, in real case). These statemets are valid throughout the paper, unless otherwise explained.

Case 2. $f=\operatorname{sn} \xi, g=\operatorname{cn} \eta+A$.

It follows from Eq. (8) that

$$
u=-\frac{2 k l \operatorname{cn} \xi \operatorname{dn} \xi \operatorname{sn} \eta \operatorname{dn} \eta}{(\operatorname{sn} \xi+\operatorname{cn} \eta+A)^{2}} .
$$

Case 3. $f=\mathrm{cn} \xi, g=\mathrm{cn} \eta+A$.

In this case, the exact elliptic function solution of Eq. (3) reads

$$
u=\frac{2 k l \operatorname{sn} \xi \operatorname{dn} \xi \operatorname{sn} \eta \operatorname{dn} \eta}{(\operatorname{cn} \xi+\operatorname{cn} \eta+A)^{2}} .
$$

In what follows, some interesting cases are considered representing the interaction of the Jacobi elliptic waves with different speeds.

Case 4. $f=\operatorname{sn}\left(k_{1} x-\omega_{1} t \mid m_{1}\right)+\operatorname{sn}\left(k_{2} x-\omega_{2} t \mid m_{2}\right) \equiv \operatorname{sn} \xi_{1}+\operatorname{sn} \xi_{2}, g=\operatorname{sn}(l y-$ $\omega t \mid m)+A \equiv \operatorname{sn} \eta+A$.

From Eq. (8), one has

$$
u=\frac{2 l\left(k_{1} \operatorname{cn} \xi_{1} \operatorname{dnn} \xi_{1}+k_{2} \operatorname{cn} \xi_{2} \operatorname{dn} \xi_{2}\right) \operatorname{cn} \eta \operatorname{dn} \eta}{\left(\operatorname{sn} \xi_{1}+\operatorname{sn} \xi_{2}+\operatorname{sn} \eta+A\right)^{2}} .
$$

Case 5. $f=\operatorname{sn} \xi_{1}+\operatorname{cn} \xi_{2}, g=\operatorname{sn} \eta+A$.

It follows from Eq. (8) that

$$
u=\frac{2 l\left(k_{1} \operatorname{cn} \xi_{1} \operatorname{dn} \xi_{1}-k_{2} \operatorname{sn} \xi_{2} \operatorname{dnn} \xi_{2}\right) \operatorname{cn} \eta \operatorname{dn} \eta}{\left(\operatorname{sn} \xi_{1}+\operatorname{cn} \xi_{2}+\operatorname{sn} \eta+A\right)^{2}} .
$$

Case 6. $f=\operatorname{cn} \xi_{1}+\operatorname{cn} \xi_{2}, g=\operatorname{sn} \eta+A$.

The elliptic function solution of Eq. (3) is

$$
u=-\frac{2 l\left(k_{1} \operatorname{sn} \xi_{1} \mathrm{dn} \xi_{1}+k_{2} \operatorname{sn} \xi_{2} \operatorname{dn} \xi_{2}\right) \operatorname{cn} \eta \operatorname{dn} \eta}{\left(\operatorname{cn} \xi_{1}+\operatorname{cn} \xi_{2}+\operatorname{sn} \eta+A\right)^{2}} .
$$

Many other cases are left to readers because of the limit of space.

\section{New types of localized coherent structures of Eq. (3)}

The long wave limit of elliptic function solutions will typically generate solitary wave forms. Such limit for the present family of elliptic function solutions is especially rich since one can proceed with the long wave limit in one direction only. Taking case 4 above as an illustrative example, we study all kinds of limit cases. Before doing this, we study the interaction property of the solution (12) by means of numerical method. Figure 1 shows the details of the interaction property of Eq. (12) with the parameters $k_{1}=1, k_{2}=2, l=1, A=4, \omega_{1}=1$, $\omega_{2}=-1, \omega=0, m_{1}=0.2, m_{2}=0.3, m=0.5$, and $t=-5,0,5$, respectively. They are valid for all the figures in this section. From the figures one can see that the wave amplitude and shape are changed after interaction. As $m_{1}$ and $m_{2} \rightarrow 1$, it 

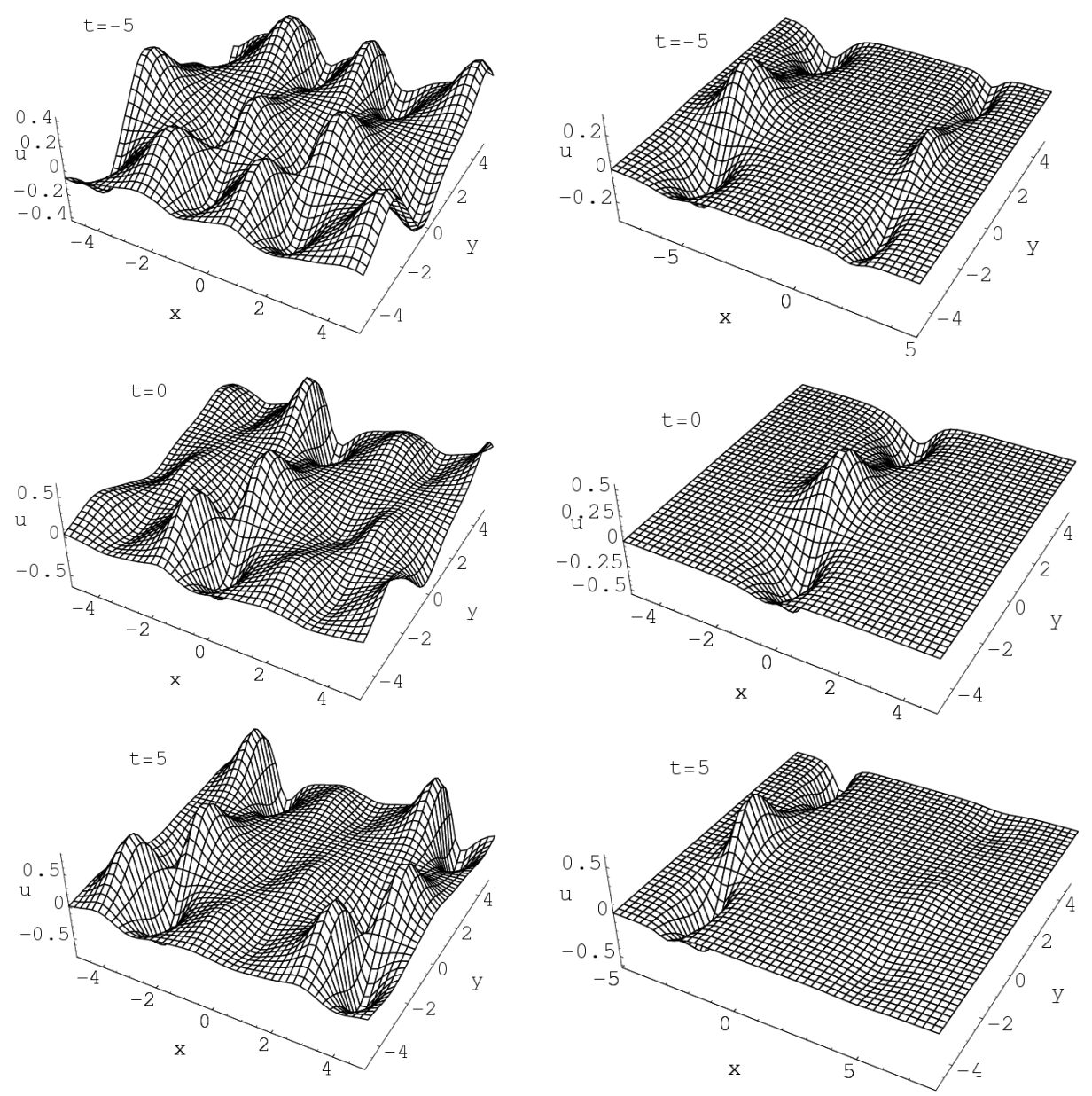

Fig. 1. The evolution of periodic wave solution (12).

Fig. 2. The evolution of Eq. (15).

follows from Eq. (12) that

$$
u=\frac{2 l\left(k_{1} \operatorname{sech}^{2} \xi_{1}+k_{2} \operatorname{sech}^{2} \xi_{2}\right) \operatorname{cn} \eta \operatorname{dn} \eta}{\left(\tanh \xi_{1}+\tanh \xi_{2}+\operatorname{sn} \eta+A\right)^{2}},
$$

whose evolution is shown in Fig. 2. It is obvious that the amplitude of periodic solitary waves is altered after interaction. When $m \rightarrow 1$, from Eq. (12) one has

$$
u=\frac{2 l\left(k_{1} \operatorname{cn} \xi_{1} \operatorname{dn} \xi_{1}+k_{2} \operatorname{cn} \xi_{2} \operatorname{dn} \xi_{2}\right) \operatorname{sech}^{2} \eta}{\left(\operatorname{sn} \xi_{1}+\operatorname{sn} \xi_{2}+\tanh \eta+A\right)^{2}} .
$$

Figure 3 illustrates the evolution of Eq. (16). It is found that the amplitude of waves has been changed after interaction. As $m_{1}, m_{2}$, and $m \rightarrow 1$, from Eq. (12) we obtain 

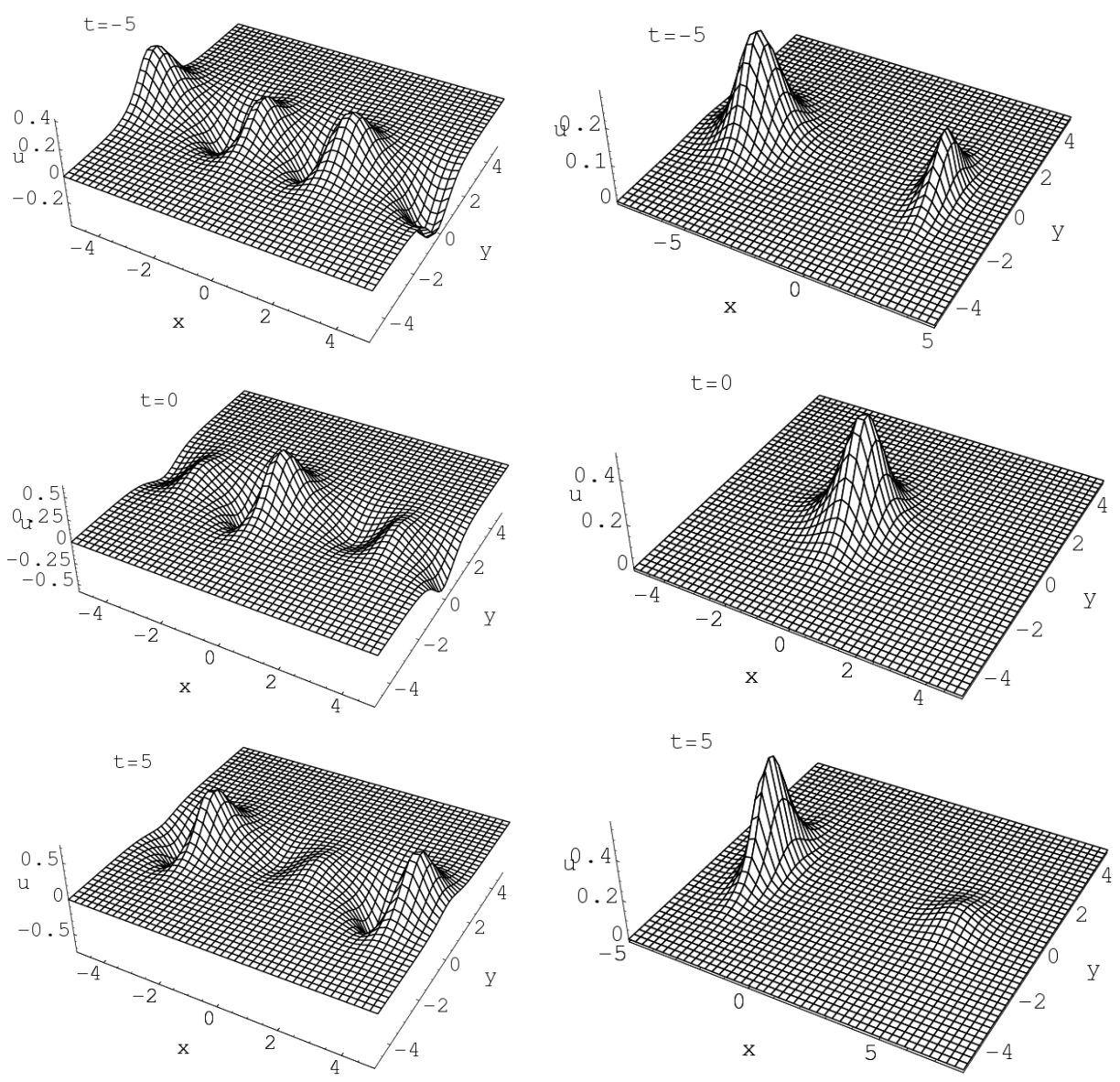

Fig. 3. The evolution of Eq. (16).

Fig. 4. The evolution of Eq. (17).

$$
u=\frac{2 l\left(k_{1} \operatorname{sech}^{2} \xi_{1}+k_{2} \operatorname{sech}^{2} \xi_{2}\right) \operatorname{sech}^{2} \eta}{\left(\tanh \xi_{1}+\tanh \xi_{2}+\tanh \eta+A\right)^{2}},
$$

whose evolution is depicted in Fig. 4. One can easily see that the interaction of two dromions is nonelastic.

\section{Conclusion and discussion}

The variable separation method has been developed for the NNV equation to obtain a general functional separation solution containing three arbitrary functions based on the singular structure analysis. By choosing these arbitrary functions to be the Jacobi elliptic functions, we have reported a series of elliptic function solutions for the equation of interest. The long wave limit of elliptic function solutions gives new types of localized coherent structures. For example, the structure of two-dromion solution (17) is quite different from that of Ref. [12]. The interac- 
tion properties of the elliptic function solution and its long wave limit cases are numerically studied.

Due to the arbitrariness of functions $f(x, t), g(y, t)$, and $\lambda(t)$ in Eq. (8), one may select them appropriately to obtain other types of exact solutions. For example, we can take these arbitrary functions as the Weierstrass elliptic functions, the square Jacobi elliptic functions, etc. All the interesting things are left to readers. It is worth noticing that the potential $v$ and $w$ in Eq. (8) may be singular because $f_{x}$ and $g_{y}$ are in the denominators. The singularity problem of solutions has been in detail discussed in Ref. [2], and is omitted here.

\section{Acknowledgments}

The author would like to express his sincere gratitude to the referees for their valuable comments. This work was supported by the Postdoctoral Science Foundation of China.

\section{References}

[1] W. Miller, Jr., in: Symmetries and Nonlinear Phenomena, Eds. D. Levi, P. Winternitz, World Scientific, London 1989, p. 188.

[2] Y.-Z. Peng, Acta Phys. Pol. A 103, 417 (2003); 105, 417 (2004); 108, 421 (2005).

[3] E.G. Kalnins, W. Miller, J. Math. Phys. 26, 1560 (1985).

[4] O.V. Kaptsov, Nonlinear Anal. 19, 753 (1992).

[5] W. Miller, Jr., L.A. Rubel, J. Phys. A 26, 1901 (1992).

[6] R.Z. Zhdanov, J. Phys. A 27, L291 (1994).

[7] E. Pucci, G. Saccomandi, Physica D 139, 28 (2000).

[8] C.Z. Qu, S.L. Zhang, R.C. Liu, Physica D 144, 97 (2000).

[9] L.P. Nizhnik, Sov. Phys. Dokl. 25, 706 (1980).

[10] S.P. Novikov, A.P. Veselov, Physica D 18, 267 (1986).

[11] M. Boiti, P.J. Leon, M. Manna, F. Pempinelli, Inverse Problems 2, 271 (1986).

[12] R. Radha, M. Lakshmanan, J. Math. Phys. 35, 4746 (1994).

[13] R. Conte, Lect. Notes Phys. 632, 1 (2003).

[14] H.C. Hu, S.Y. Lou, Q.P. Liu, arXiv: nlin. SI/0210012 v1 7 Oct 2002.

[15] M. Abramowitz, I.A. Stegun, Handbook of Mathematical Functions, Dover, New York 1972. 